\title{
Parametric Macromodeling using Interpolation of Sylvester based State-Space Realizations
}

\author{
Elizabeth Rita Samuel ${ }^{1}$, Luc Knockaert ${ }^{1}$ and Tom Dhaene ${ }^{1}$ \\ Department of Information Technology, Ghent University, Gaston Crommenlaan 8 Bus 201, B-9050 Gent, Belgium ${ }^{1}$ \\ esamuel@intec.ugent.be,luc.knockaert@intec.ugent.be,tom.dhaene@intec.ugent.be
}

\begin{abstract}
Keywords: Sylvester equation: parametric macromodeling: state-space matrices: rational approximation: interpolation.
Abstract: A novel state-space realization for parametric macromodeling is proposed in this paper. A judicious choice of the state-space realization is required to account for the generally assumed smoothness of the state-space matrices with respect to the design parameters. This is used in combination with suitable interpolation schemes to interpolate a set of state-space matrices, and hence the poles and residues indirectly, in order to build accurate parametric macromodels. The key points are the choice of a proper pivot matrix and the solution of a Sylvester equation for pole placement. Pertinent numerical examples validate the proposed state-space realization for parametric macromodeling.
\end{abstract}

\section{INTRODUCTION}

When designing a system, or implementing a controller to augment to an existing system the basic step is to obtain a mathematical model. For this, design space exploration, design optimization, and sensitivity analysis are usually performed and this requires multiple simulations for different design parameter values (e.g., layout features). Parametric macromodeling is an efficient and accurate tool to perform these design activities, while avoiding new measurements or simulations for each new parameter configuration. Parametric macromodels are multivariate models that describe the complex behavior of the systems, typically characterized by frequency (or time) and several geometrical and physical design parameters, such as layout or substrate features. Recently, parametric macromodeling techniques which are able to guarantee overall stability and passivity have been proposed in (Ferranti et al., 2010a; Deschrijver and Dhaene, 2008; Ferranti et al., 2009; Ferranti et al., 2010b; Triverio et al., 2010). The techniques described in (Ferranti et al., 2010a) and (Ferranti et al., 2009) are based on the interpolation of a set of univariate macromodels, called root macromodels. This interpolation process of input-output systems leads to parameterization of the residues, but unfortunately not of the poles. Passive interpolation of the state-space matrices of a set of root macromodels is proposed in (Ferranti et al., 2010b) and(Triverio et al., 2010), providing an increased modeling capability with re- spect to (Ferranti et al., 2010a) and (Ferranti et al., 2009), due to the parameterization of both poles and residues. Unfortunately, these methods are sensitive to issues related to the interpolation of state-space matrices (De Caigny et al., 2009), such as the smoothness of the state-space matrices as a function of the parameters.

In this paper, we propose a novel state-space realization that is suitable to build accurate parametric macromodels. The direct parameterization of poles and residues is avoided, due to their potentially nonsmooth effect with respect to the design parameters. A conversion from a pole-residue form obtained by means of Vector Fitting (VF) (Gustavsen and Semlyen, 1999) to a Sylvester realization is computed for each root macromodel. Since the same pivot (reference) matrix is used for all state-space realizations of the root macromodels, smooth variations of the statespace matrices with respect to the design parameters are expected. It leads to build accurate parametric macromodels using suitable interpolation schemes.

We focus on applications described by scattering (S) parameters, but the approach can be also used for other system representations, e.g. admittance and impedance parameters. Pertinent numerical examples validate the proposed state-space realization for macromodeling. 


\section{PARAMETRIC MACROMODELING}

Starting from a set of $M$ data samples $\left\{(s, \vec{p})_{k}, H(s, \vec{p})_{k}\right\}_{k=1}^{M}$, a set of frequency-dependent rational models is built for some design space points by means of system identification techniques (Pintelon et al., 1994), in our case the VF technique (Gustavsen and Semlyen, 1999). The result of this initial procedure is a set of rational univariate macromodels with Gilbert realization, called root macromodels. Each root macromodel is related to a root point $\vec{p}=\left(p_{k_{1}}^{(1)}, \ldots, p_{k_{M}}^{(M)}\right)$ in the design space. Two data grids are used in the modeling process: an estimation grid and a validation grid. The first one is utilized to build the root macromodel which, combined with an interpolation scheme, provide the parametric macromodel. The second grid, more dense than the previous one, is utilized to assess the interpolation capability of the parametric macromodel, its capability of describing the system under study in points of the design space previously not used for the construction of the root macromodel.

Suppose we have a set of models $S_{\vec{p}_{k}}, k=$ $1, \ldots, N$ with given minimal realizations

$$
\mathcal{S}_{\vec{p}_{k}} \equiv\left[\begin{array}{cc}
A_{\vec{p}_{k}} & B_{\vec{p}_{k}} \\
C_{\vec{p}_{k}} & D_{\vec{p}_{k}}
\end{array}\right],
$$

state-space equations

$$
\begin{aligned}
\dot{x} & =A_{\vec{p}_{k}} x+B_{\vec{p}_{k}} u \\
y & =C_{\vec{p}_{k}} x+D_{\vec{p}_{k}} u
\end{aligned}
$$

and transfer functions

$$
R_{\vec{p}_{k}}(s)=C_{\vec{p}_{k}}\left(s I-A_{\vec{p}_{k}}\right)^{-1} B_{\vec{p}_{k}}+D_{\vec{p}_{k}}
$$

In this paper we suppose that all realizations $S_{\vec{p}_{k}}$ have the same McMillan degree $N$ and number of ports $P \leq N$. We further suppose that all matrices $A_{\vec{p}_{k}}$ are Hurwitz stable.

We propose a generic parametric realization of the form

$$
\mathcal{S}(\vec{p}) \equiv\left[\begin{array}{ll}
A(\vec{p}) & B(\vec{p}) \\
C(\vec{p}) & D(\vec{p})
\end{array}\right]
$$

with $\vec{p}=\left(\vec{p}^{(1)}, \ldots, \vec{p}^{(M)}\right)$. The models $S_{\vec{p}_{k}}$ can be considered as snapshots of $\mathcal{S}(\vec{p})$ generated by fixing the parameter $\vec{p}$ at the fixed values $\vec{p}_{k}$. $\mathcal{S}_{\vec{p}_{k}}$ must be able to accurately model the system behavior as a function of $s$ and $\vec{p}$.

\section{STATE-SPACE REALIZATION}

To obtain accurate parametric macromodels by interpolating the state-space matrices, the realization is very important.
In what follows, we discuss the well-known Gilbert and Balanced realizations, and the proposed novel Sylvester realization.

\subsection{Gilbert Realization}

The minimal state-space realization problem for linear time invariant (LTI) systems was first stated by Gilbert (Gilbert, 1963), who gave an algorithm for transforming a transfer function into a system of differential equations (i.e. a state-space description). The approach of Gilbert is based on partial-fraction expansions. In the Gilbert approach the poles and the residues are stamped directly in the state-space matrices and since poles and residues may present a highly non-smooth behavior with respect to design parameters, achieving a high accuracy in parametric macromodels built by interpolation of state-space matrices becomes difficult.

\subsection{Balanced Realization}

A minimal and stable realization is called balanced (Moore, 1981; Pernebo and Silverman, 1982) if the controllability and observability Gramians are equal and diagonal. Every minimal system can be brought into balanced form. The balanced realization can be implemented using the Matlab function balreal. This routine uses the eigen decomposition of the product of the observability and controllability Gramians to construct the balancing transformation matrix. As stated in (De Caigny et al., 2009; Peeters et al., 2009), uniqueness is guaranteed up to a sign and it may effect the smoothness of the state-space matrices as functions of design parameters.

The problem with the interpolation procedure for the Gilbert and balanced realization is that, although the interpolation technique yields (by construction) the discrete macro-model $S_{\vec{p}_{k}}$ for $\vec{p}=\vec{p}_{k}$, it is not at all sure that the interpolated matrices $A(\vec{p}), B(\vec{p}), C(\vec{p}), D(\vec{p})$ will behave smoothly between the nodes $p_{k}$. The reason for this is that minimal realizations are all equivalent modulo a similarity transformation, i.e., two realizations related by

$$
\left[\begin{array}{cc}
\tilde{A}_{\vec{p}_{k}} & \tilde{B}_{\vec{p}_{k}} \\
\tilde{C}_{\vec{p}_{k}} & \tilde{D}_{\vec{p}_{k}}
\end{array}\right]=\left[\begin{array}{cc}
X^{-1} A_{\vec{p}_{k}} X & X^{-1} B_{\vec{p}_{k}} \\
C_{\vec{p}_{k}} X & D_{\vec{p}_{k}}
\end{array}\right]
$$

where $X$ is any nonsingular matrix, yield the same transfer function

$$
\begin{aligned}
H(s) & =C_{\vec{p}_{k}}\left(s I-A_{\vec{p}_{k}}\right)^{-1} B_{\vec{p}_{k}}+D_{\vec{p}_{k}} \\
& =\tilde{C}_{\vec{p}_{k}}\left(s I-\tilde{A}_{\vec{p}_{k}}\right)^{-1} \tilde{B}_{\vec{p}_{k}}+\tilde{D}_{\vec{p}_{k}}
\end{aligned}
$$

It is important to note that the interpolation of state-space matrices allows a higher modeling capa- 
bility than the interpolation of transfer functions (Ferranti et al., 2010a; Ferranti et al., 2009), but unfortunately these methods are sensitive to issues related to the smoothness of the state-space matrices as a function of the parameters. In the following subsection, the novel realization can guarantee smoothness since they are pivot-based realizations.

\subsection{Sylvester Realization}

We propose the following state-space feedback realization with feedback matrix $F$ and pivot matrix $\tilde{A}$

$$
\begin{aligned}
\dot{x} & =\tilde{A} x+\tilde{B}_{\vec{p}_{k}} v \\
y & =\tilde{C}_{\vec{p}_{k}} x+\tilde{D}_{\vec{p}_{k}} v \\
v & =-F x+u
\end{aligned}
$$

where $\tilde{A}$ is a fixed $N \times N$ pivot matrix and $F$ is a fixed $p \times N$ state-space feedback matrix. This realization can be written as

$$
\mathcal{R}_{\vec{p}_{k}} \equiv\left[\begin{array}{cc}
\tilde{A}-\tilde{B}_{\vec{p}_{k}} F & \tilde{B}_{\vec{p}_{k}} \\
\tilde{C}_{\vec{p}_{k}}-\tilde{D}_{\vec{p}_{k}} F & \tilde{D}_{\vec{p}_{k}}
\end{array}\right]
$$

For $\mathcal{R}_{\vec{p}_{k}}$ and $\mathcal{S}_{\vec{p}_{k}}$ to be equivalent, the existence of nonsingular matrices $X_{k}$ such that

$$
\begin{aligned}
\tilde{A}-\tilde{B}_{\vec{p}_{k}} F & =X_{k}^{-1} A_{\vec{p}_{k}} X_{k} \\
\tilde{B}_{\vec{p}_{k}} & =X_{k}^{-1} B_{\vec{p}_{k}} \\
\tilde{C}_{\vec{p}_{k}} & =C_{\vec{p}_{k}} X_{k}
\end{aligned}
$$

is needed.

By eliminating (10b) from (10a) we obtain the Sylvester equation

$$
A_{\vec{p}_{k}} X_{k}-X_{k} \tilde{A}+B_{\vec{p}_{k}} F=0
$$

for the unknown matrix $X_{k}$. We need the following

Theorem 1. The Sylvester equation (11) has a unique nonsingular solution $X_{k}$ provided the pair $\left(A_{\vec{p}_{k}}, B_{\vec{p}_{k}}\right)$ is controllable, the pair $(\tilde{A}, F)$ is observable, and the intersection of the eigenspectra of $A_{\vec{p}_{k}}$ and $\tilde{A}$ is empty.

Proof. See (de Souza and Bhattacharyya, 1981; Varga, 2000).

Note that Sylvester equations are routinely solved by the Matlab function lyap.

Remark 1. The Sylvester realizations given the pivot matrix $\tilde{A}$ and feedback matrix $F$, are all unique by construction. For the choice of $\tilde{A}$ we can take a blockdiagonal or block-Jordan matrix(Varga, 2000) which never shares eigenvalues with any of the $A_{\vec{p}_{k}}$ matrices.
This can be accomplished by choosing the eigenvalues of A close to the imaginary axis (see also the numerical simulations). The choice of $F$ is subject to the requirement that the pair $(\tilde{A}, F)$ has to be observable. In some cases such as the Gilbert (Gilbert, 1963) or Vector Fitting (Gustavsen and Semlyen, 1999) realization, all matrices $B_{k}$ are equal, and then a judicious choice for $F$ is $F=B_{k}^{T}$. In the Appendix we show that, under very general conditions, there exist realizations such that all $B_{k}$ are equal. More generally speaking, $F$ can be chosen quite freely, or its choice can be imbedded in the overall Sylvester algorithm (Carvalho et al., 2003).

\section{NUMERICAL EXAMPLES}

In the following examples, we show the importance of the realization issue, and validate the proposed Sylvester approach, by comparing them with the standard Gilbert and balanced realizations.

\subsection{Two coupled microstrip with Variable Length (CM)}

Two coupled microstrips can be modeled (Knockaert and De Zutter, 2000) starting from per-unit-length parameters. The cross section is shown in Figure.1.

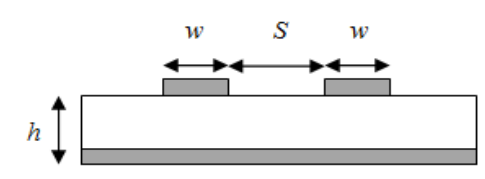

Figure 1: CM: Two coupled microstrip line.

Figure. 1 shows its cross section. The length $L$ are considered as variable parameters in addition to frequency. Their corresponding ranges are shown in Table 1 .

Table 1: CM: PARAMETERS OF THE COUPLED MICROSTRIPS

\begin{tabular}{|l|l|l|}
\hline Parameter & Min & Max \\
\hline Frequency $($ freq) & $20 \mathrm{MHz}$ & $8 \mathrm{GHz}$ \\
Length $(L)$ & $2.5 \mathrm{~cm}$ & $3 \mathrm{~cm}$ \\
\hline
\end{tabular}

The scattering parameters were obtained over a validation grid of $200 \times 11$ samples, for frequency and length respectively. We have built root macromodels for 6 values of the spacing by means of VF, each with an order 11 . 
As described in Section 3.3, a pivot matrix and a feedback matrix is chosen such that a wellconditioned solution is obtained for the Sylvester equation (11). We use a VF form of pivot matrix (Gustavsen, ), which can be coded in Matlab as

$\mathrm{N} ; \%$ Order of approximation

oComplex conjugate pairs, linearly

spaced:

bet=linspace (w(1), w (end), N/2) ;

poles $=[]$;

for $\mathrm{n}=1$ : length (bet)

alpha $=-\operatorname{beta}(n) * 1 e-2$;

poles $=[$ poles $($ alpha-i*beta $(n))$

(alpha+i*beta $(\mathrm{n}))$ ] ;

end

i.e; the pole pairs are chosen as

$$
a_{n}=-\alpha+j \beta, \quad a_{n}=-\alpha-j \beta
$$

where, $\alpha=\beta / 100$.

Also, since the eigenvalues of the pivot matrix and those of the root macromodels obtained from Gilbert realization must not be the same, we choose the poles very close to the imaginary axis as shown in Figure.2. The feedback matrix is chosen as column

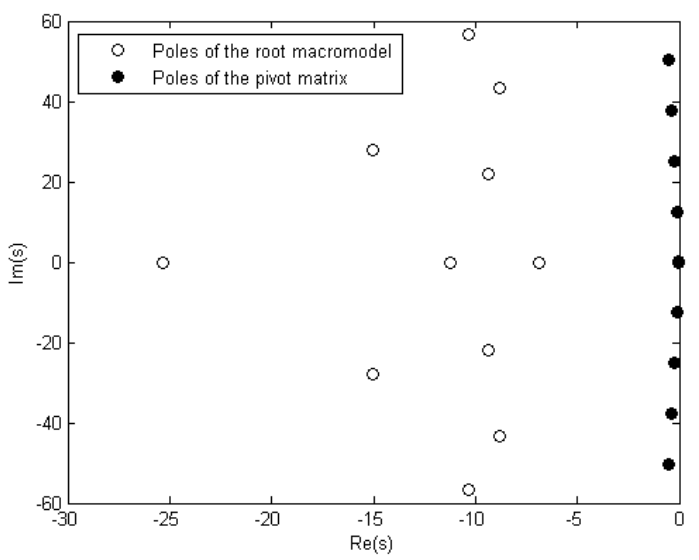

Figure 2: CM: Eigenvalues of the pivot matrix and the root macromodels obtained from Gilbert realization.

vectors of 1's, 2's and 0's similar to VF technique. A similarity transformation is then performed using the Sylvester solution to obtain the state-space matrices of the Sylvester realization.

Finally, a bivariate macromodel is obtained by linear interpolation of the corresponding state-space matrices using the Sylvester realization as shown in Figure.3.

The maximum absolute error over the validation grid for the parametric macromodel of the scattering

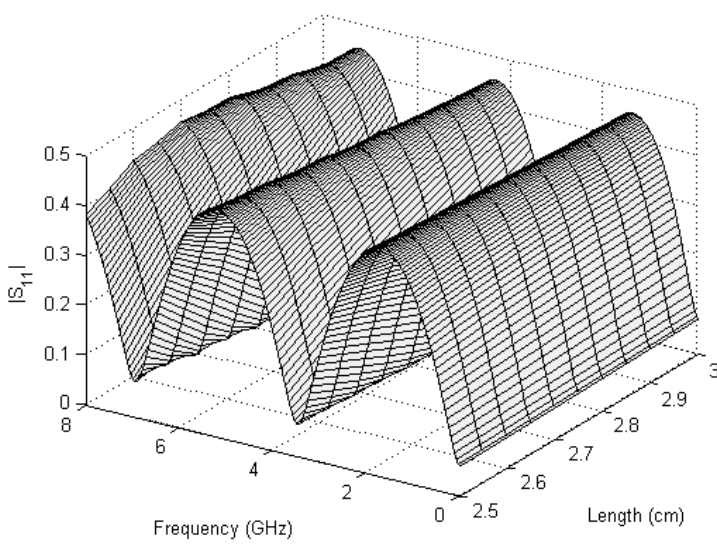

Figure 3: CM: Magnitude of the bivariate macromodel $S_{11}(s, L)$ (Sylvester realization for each root macromodel).

matrix is bounded by $-56 \mathrm{~dB}$. It can be noted that a very good agreement is obtained between the original data and the proposed parametric macromodeling technique. The parametric macromodel captures the behavior of the system very accurately over the entire range of the length.

Figure. 4 shows that direct parameterization of the poles should be avoided due to potentially nonsmooth behavior with respect to the design parameters with Gilbert realization.



Figure 4: CM: Magnitude of the bivariate macromodel $S_{11}(s, L)$ (Gilbert realization form for each root macromodel).

In Figure. 5 it is shown that the maximum absolute error is very small for the Sylvester but it is not satisfactory for the Gilbert and balanced real realization. 


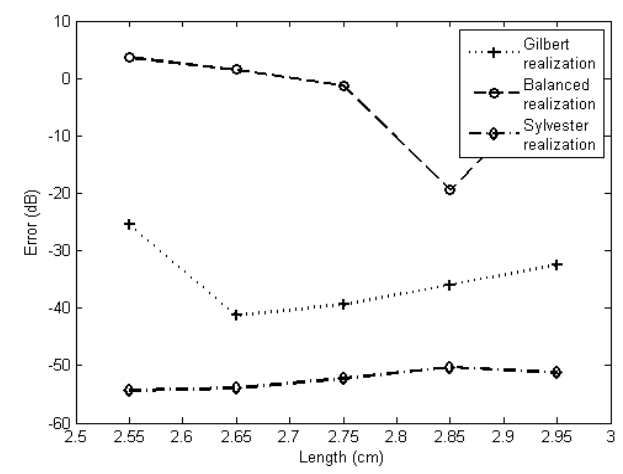

Figure 5: CM: Absolute error comparison for the different realizations.

\subsection{Hairpin bandpass microwave filter (HP)}

In this example, a hairpin bandpass filter with the layout shown in Figure. 6 is modeled. The relative permittivity of the substrate is 9.9, and its thickness is equal to $0.635 \mathrm{~mm}$.

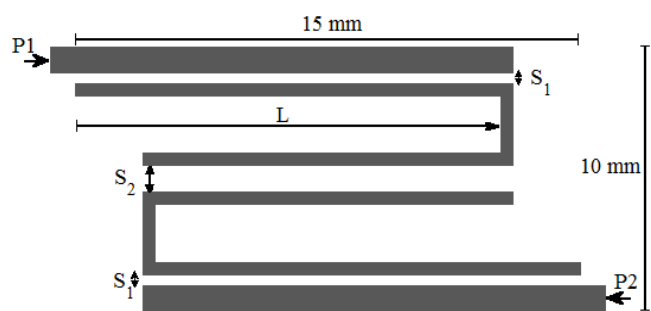

Figure 6: HP: Layout of the Hairpin bandpass microwave filter.

The spacing $S_{1}$ and the length $L$ of the stub are chosen as design variables in addition to frequency. Their corresponding ranges are shown in Table 2.

Table 2: HP: PARAMETERS OF THE HAIRPIN BANDPASS MICROWAVE FILTER

\begin{tabular}{|l|l|l|}
\hline Parameter & Min & Max \\
\hline Frequency $($ freq $)$ & $1.5 \mathrm{GHz}$ & $3.5 \mathrm{GHz}$ \\
Length $(L)$ & $12 \mathrm{~mm}$ & $12.5 \mathrm{~mm}$ \\
Spacing $\left(S_{1}\right)$ & $0.27 \mathrm{~mm}$ & $0.32 \mathrm{~mm}$ \\
\hline
\end{tabular}

The scattering parameters have been computed by means of the advanced design system (ADS) over a grid of $11 \times 7$ samples, for length and spacing respectively. We have built root macromodels for $6 \times 4$ values of the length and spacing respectively by means of VF, each with an order 13. Next the realization approaches as described in Section 3.3 is used to obtain Sylvester realized state-space form for each root macromodel. Finally, a trivariate macromodel is obtained by multilinear interpolation of the corresponding state-space matrices as shown in Figure.7.

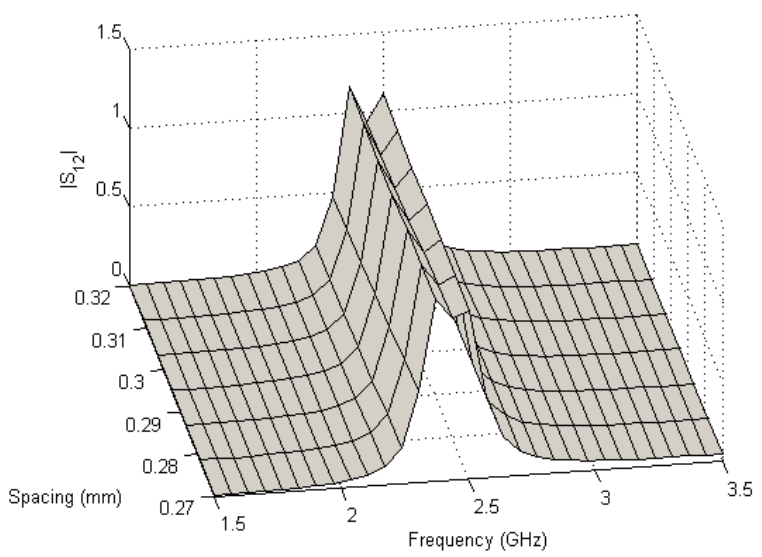

Figure 7: HP: Magnitude of the trivariate macromodel $S_{12}(s, L, S)$ for $L=12.05 \mathrm{~mm}$ (Sylvester realization for each root macromodel).

The maximum absolute error over the validation grid for the parametric macromodel of the scattering matrix is bounded by $-58 \mathrm{~dB}$. It can be noted that a very good agreement is obtained between the original data and the proposed parametric macromodeling technique. The parametric macromodel captures the behavior of the system very accurately over the entire design space.

Figure. 8 shows the parametric macromodel using balanced real realization. It is seen by comparing with Figure.7 that the behavior is very erratic.

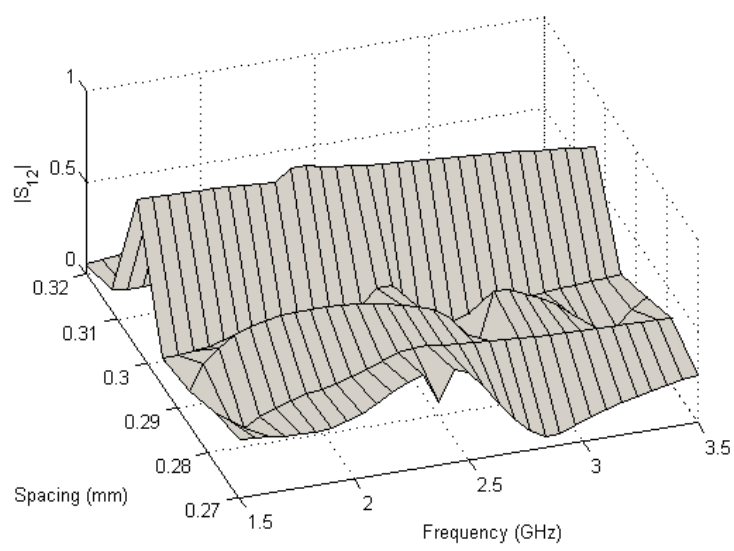

Figure 8: HP: Magnitude of the trivariate macromodel $S_{12}(s, L, S)$ for $L=12.05 \mathrm{~mm}$ (Balanced realization for each root macromodel).

For the hairpin filter it can be also noted from 
the Figure. 9 that the maximum absolute error is very small for the Sylvester realization but it is not satisfactory for Gilbert realization and balanced real realization.

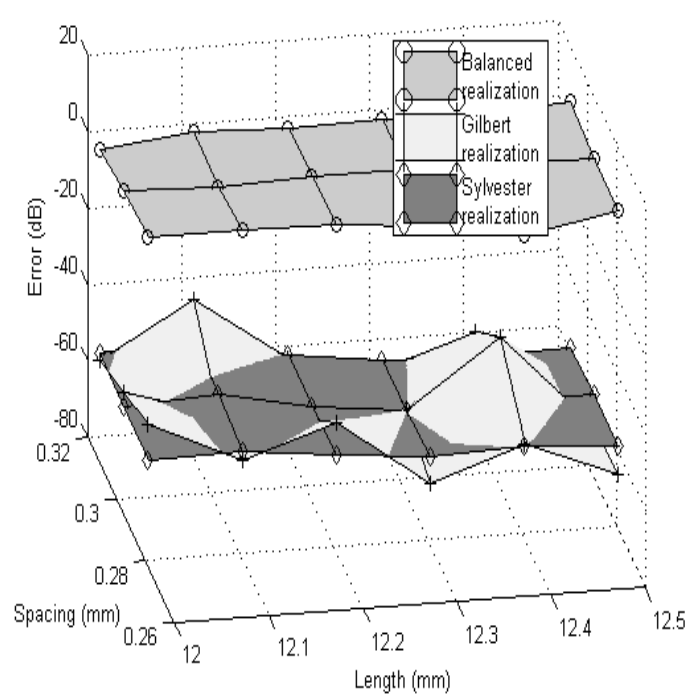

Figure 9: HP: Absolute error comparison for the different realizations.

\section{CONCLUSIONS}

This paper proposes a novel state-space realization for parametric macromodeling. For the generally assumed smoothness of the state-space matrices with respect to the parameters a wise choice of the state space realization is required. This realization is used in combination with suitable interpolation schemes to interpolate the set of state-space matrices in order to build accurate parametric macromodels. The key point is to find a suitable pivot matrix and to solve Sylvester equations such that well conditioned solution are obtained. From the numerical examples it is seen that the proposed realization technique generates a more accurate parametric model with respect to the design parameters in comparison to the Gilbert realization and balanced realization.

\section{ACKNOWLEDGEMENTS}

This work was supported by the Research Foundation Flanders (FWO) and by the Interuniversity Attraction Poles Programme BESTCOM initiated by the Belgian Science Policy Office.

\section{REFERENCES}

Carvalho, J., Datta, K., and Hong, Y. (2003). A new block algorithm for full-rank solution of the sylvesterobserver equation. IEEE Transactions on Automatic Control, 48(12):2223 - 2228.

De Caigny, J., Camino, J. F., and Swevers, J. (2009). Interpolating model identi- cation for siso linear parametervarying systems. Mechanical Systems and Signal Processing, 23(8):2395-2417.

de Souza, E. and Bhattacharyya, S. (1981). Controllability, observability and the solution of AX $-\mathrm{XB}=\mathrm{C}$. Linear Algebra and its Applications, 39:167 - 188.

Deschrijver, D. and Dhaene, T. (2008). Stability and passivity enforcement of parametric macromodels in time and frequency domain. IEEE Transactions on Microwave Theory and Techniques, 56(11):2435 -2441.

Ferranti, F., Knockaert, L., and Dhaene, T. (2009). Parameterized S-parameter based macromodeling with guaranteed passivity. IEEE Microwave and Wireless Component Letters, 19(10):608-610.

Ferranti, F., Knockaert, L., and Dhaene, T. (2010a). Guaranteed passive parameterized admittance-based macromodeling. IEEE Transactions on Advanced Packaging, 33(3):623-629.

Ferranti, F., Knockaert, L., Dhaene, T., and Antonini, G. (2010b). Passivity-preserving parametric macromodeling for highly dynamic tabulated data based on Lur'e equations. IEEE Transactions on Microwave Theory and Techniques, 58(12):3688 -3696.

Gilbert, E. G. (1963). Controllability and observability in multi-variable control systems. SIAM Journal on Control, 1(2):128-151.

Gustavsen, B. USERS GUIDE FOR vectfit3.m (Fast, Relaxed Vector Fitting) for Matlab.

Gustavsen, B. and Semlyen, A. (1999). Rational approximation of frequency domain responses by vector fitting. IEEE Transaction on Power Delivery, 14:10521061.

Knockaert, L. and De Zutter, D. (2000). Laguerre-SVD reduced-order modeling. IEEE Transactions on Microwave Theory and Techniques, 48(9):1469-1475.

Moore, B. (1981). Principal component analysis in linear systems: Controllability, observability, and model reduction. IEEE Transactions Automatic Control, 26(1):17-31.

Peeters, R., Olivi, M., and Hanzon, B. (2009). Balanced realization of lossless systems: Schur parameters, canonical forms and applications. 15th IFAC Symposium on System Identification, pages 273-283.

Pernebo, L. and Silverman, L. M. (1982). Model reduction via balanced state space representations. IEEE Transactions Automatic Control, 27(2):382-387.

Pintelon, R., Guillaume, P., and Rolain, Y. (1994). Parametric identification of transfer functions in the frequency domain- A survey. IEEE Transactions on Automatic Control, 39(11):2245-2260.

Triverio, P., Nakhla, M., and Grivet-Talocia, S. (2010). Passive parametric modeling of interconnects and packaging components from sampled impedance, admit- 
tance or scattering data. Electronic System-Integration Technology Conference, pages 1-6.

Varga, A. (2000). Robust pole assignment via sylvester equation based state feedback parametrization. Proceedings of the IEEE International Symposium on Computer-Aided Control System Design, pages 1318.

\section{APPENDIX}

We need to prove that, given a matrix $\tilde{B}$, there exists a nonsingular matrix $T_{k}$ such that $T_{k}^{-1} B_{k}=\tilde{B}$. The resulting equivalent realization is then

$$
\left[\begin{array}{cc}
T_{k}^{-1} A_{k} T_{k} & \tilde{B} \\
{ }^{k} T_{k} & D_{k}
\end{array}\right]
$$

This is proved under quite general circumstances by the following theorem :

Theorem 2. Let $n \geq m$ and $B, \tilde{B} n \times m$ full rank $m a$ trices. Then the matrix equation $T \tilde{B}=B$ admits $a$ nonsingular $n \times n$ matrix solution $T$.

Proof. First suppose $n=m$. Then $T=B \tilde{B}^{-1}$ is nonsingular. Next suppose $m<n$. Consider the QR decompositions of $B$ and $\tilde{B}$ :

$$
B=Q\left[\begin{array}{l}
R \\
0
\end{array}\right] \quad \tilde{B}=Q_{1}\left[\begin{array}{c}
R_{1} \\
0
\end{array}\right]
$$

Then the matrix

$$
T=Q\left[\begin{array}{cc}
R R_{1}^{-1} & 0 \\
0 & Y
\end{array}\right] Q_{1}^{T}
$$

with $Y$ nonsingular, is itself nonsingular, and satisfies

$$
T \tilde{B}=Q\left[\begin{array}{l}
R \\
0
\end{array}\right]=B
$$

The proof is complete. Note that for simplicity we can take $Y=I_{n-m}$. 